\title{
A Comparison of Cooling Techniques to Treat Cardiac Arrest Patients with Hypothermia
}

\author{
Anna Finley Caulfield, ${ }^{1}$ Shylaja Rachabattula, ${ }^{1}$ Irina Eyngorn, ${ }^{1}$ Scott A. Hamilton, ${ }^{1}$ \\ Rajalakshmi Kalimuthu, ${ }^{1}$ Amie W. Hsia, ${ }^{2}$ Maarten G. Lansberg, ${ }^{1}$ \\ Chitra Venkatasubramanian, ${ }^{1}$ J. J. Baumann, ${ }^{1}$ Marion S. Buckwalter, ${ }^{1}$ Monisha A. Kumar, ${ }^{3}$ \\ James S. Castle, ${ }^{4}$ and Christine A. C. Wijman ${ }^{1}$
}

\author{
${ }^{1}$ Stanford Neurocritical Care Program, Stanford Stroke Center, Stanford University School of Medicine Center, Palo Alto, \\ CA 94304, USA \\ ${ }^{2}$ Stroke Washington Hospital Center, Washington, DC 20010, USA \\ ${ }^{3}$ Department of Neurology, Hospital of the University of Pennsylvania, Philadelphia, PA 19104, USA \\ ${ }^{4}$ Department of Neurology, NorthShore University Health System, The University of Chicago, Evanston, IL 60201, USA
}

Correspondence should be addressed to Anna Finley Caulfield, afinley@stanford.edu

Received 17 December 2010; Accepted 24 March 2011

Academic Editor: Thomas M. Hemmen

Copyright (c) 2011 Anna Finley Caulfield et al. This is an open access article distributed under the Creative Commons Attribution License, which permits unrestricted use, distribution, and reproduction in any medium, provided the original work is properly cited.

\begin{abstract}
Introduction. We sought to compare the performance of endovascular cooling to conventional surface cooling after cardiac arrest. Methods. Patients in coma following cardiopulmonary resuscitation were cooled with an endovascular cooling catheter or with ice bags and cold-water-circulating cooling blankets to a target temperature of $32.0-34.0^{\circ} \mathrm{C}$ for 24 hours. Performance of cooling techniques was compared by (1) number of hourly recordings in target temperature range, (2) time elapsed from the written order to initiate cooling and target temperature, and (3) adverse events during the first week. Results. Median time in target temperature range was 19 hours (interquartile range (IQR), 16-20) in the endovascular group versus. 10 hours (IQR, 7-15) in the surface group $(P=.001)$. Median time to target temperature was 4 (IQR, 2.8-6.2) and 4.5 (IQR, 3-6.5) hours, respectively $(P=.67)$. Adverse events were similar. Conclusion. Endovascular cooling maintains target temperatures better than conventional surface cooling.
\end{abstract}

\section{Introduction}

Each year an estimated 165,000 people in the United States have an out-of-hospital cardiac arrest, and many more undergo in-hospital resuscitation [1-4]. Those that survive may have devastating neurological impairments from global ischemic brain injury. Mild-to-moderate therapeutic hypothermia for 12 to 24 hours has been shown to improve neurological outcome in two randomized clinical trials of comatose survivors following out-of-hospital ventricular fibrillation arrest $[5,6]$. Based on the results of these trials, the 2005 American Heart Association guidelines for Cardiopulmonary Resuscitation and Emergency Cardiovascular Care recommended to consider the use of therapeutic hypothermia for unconscious adult patients with return of spontaneous circulation after witnessed out-of-hospital ventricular fibrillation arrest (Class IIA) and nonventricular fibrillation and in-hospital cardiac arrest (Class IIB) [7]. The 2010 guidelines continue to recommend therapeutic hypothermia in these patients, now as Class I and Class IIB recommendations, respectively [8].

In spite of the data and guidelines hospitals in the United States have been slow to adopt therapeutic hypothermia in the routine management of comatose postcardiac arrest patients [9]. This may be in part explained by physician unfamiliarity with therapeutic hypothermia and in part by the labor intensiveness and inaccuracy of surface cooling using ice bags and cooling blankets [10]. Most studies to date have used conventional surface-based cooling techniques (ice bags and cool air or water blankets), which are generally 


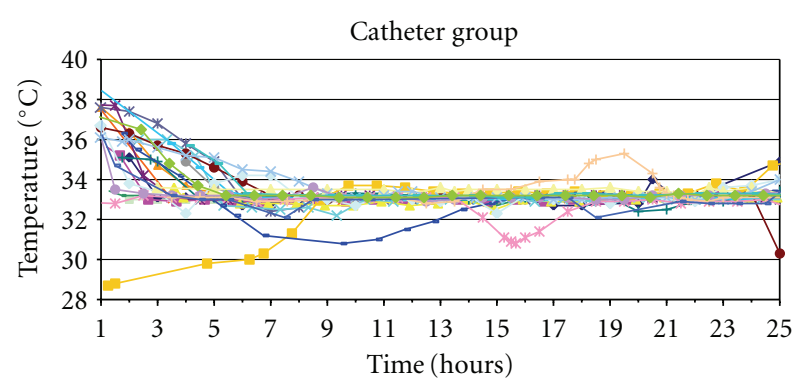

(a)

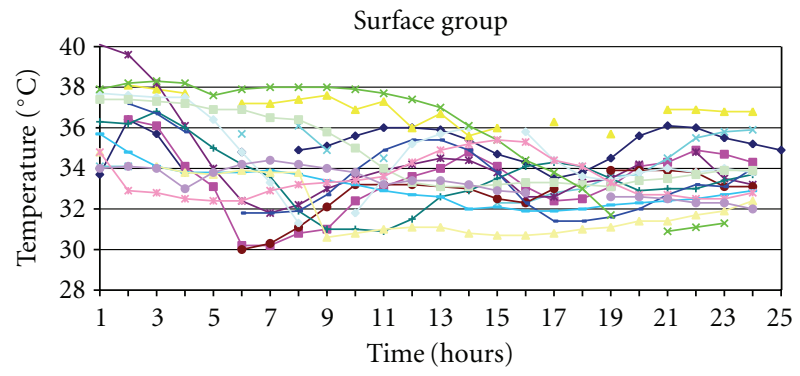

(b)

FIGURE 1: Hourly bladder temperature recordings of each patient from the time point that the cooling protocol was initiated to the end of active cooling (24 hours). (a) Endovascular-cooled group $(n=26)$. (b) Surface-cooled group $(n=15)$.

slow and imprecise in achieving and maintaining target temperature. Animal models suggest that a delay in cooling abates the neurological benefits of mild hypothermia and that deep hypothermia has no added benefit over mildto-moderate hypothermia $[11,12]$. Endovascular cooling techniques have shown promise in providing more rapid and precise temperature regulation than conventional surface cooling techniques and have been shown to be feasible in postcardiac arrest patients, but data on their utility is scarce [13-16]. The purpose of this study is to compare the performance of endovascular versus conventional surfacebased cooling in comatose postcardiac arrest patients.

\section{Materials and Methods}

This was a longitudinal comparative study of consecutive comatose postcardiac arrest survivors, who were treated with our institution's hypothermia protocol (see the appendix). All patients were sedated and paralyzed to ensure comfort and prevent shivering. Target core temperature measured by bladder temperature was $32.0-34.0^{\circ} \mathrm{C}$ for 24 hours followed by spontaneous or passive rewarming over 12 hours. The start of the 24-hour cooling period was designated as the time of the written order to initiate the therapeutic hypothermia protocol and included the organizational time required for setting up the surface or endovascular cooling equipment and for the catheter insertion. All patients had bladder temperatures recorded hourly from the initiation of the cooling protocol. This study was approved by our institutional review board.
The study spanned the time period between February 2004 and September 2006 ( 2.5 years). Between February 2004 and February 2005, hypothermia was induced using cold-water-circulating cooling blankets (Mul-T-Blanket with Gaymar Medi Therm III, Gaymar Industries, Orchard Park, NY) and ice bags. In February 2005 our institution converted to endovascular cooling using the Celsius Control System (Innercool Therapies, San Diego, CA) catheter. This catheter system has a feedback loop controlling target temperature by using a temperature sensing esophageal probe. In patients who underwent endovascular cooling, surface cooling was initiated until the catheter could be inserted. Patients did not receive ice-cooled intravenous fluids. The catheter was removed at the end of the 24-hour cooling period, and spontaneous or passive rewarming occurred over 12 hours.

The performance of endovascular versus surface-based cooling was compared by assessing the following variables: (1) number of hourly recordings in target temperature range $\left(32-34^{\circ} \mathrm{C}\right)$ during the 24 -hour cooling period, (2) time elapsed from the written orders to initiate the cooling protocol and the target temperature achieved (time required to insert the catheter was included in this time period), and (3) frequency of predefined adverse events possibly related to hypothermia or the use of an endovascular catheter or surface cooling technique during the first 7 days after cardiac arrest. Data was collected using the patients' medical records. Details of the diagnostic criteria for adverse events may be found at the bottom of Table 1 .

2.1. Statistical Analysis. Continuous variables were analyzed with the Wilcoxon Rank-Sum test. Categorical variables were analyzed with the Fisher's exact test. Group differences were considered significant at $P<.05$.

\section{Results}

Forty-one cardiac arrest patients underwent hypothermia between February 2004 and September 2006 at our hospital: 15 with surface and 26 with endovascular cooling. In five patients in whom hypothermia was considered using endovascular cooling, surface cooling was used instead, because of a failed attempt at catheter placement $(N=1)$, a contraindication to catheter placement $(N=2)$ or the general ICU team preferred surface cooling $(N=2)$. There were no differences in baseline characteristics between the two groups except for a higher proportion of patients with ventricular fibrillation arrest in the endovascular group and a strong trend towards a higher proportion of renal failure on admission in the surface cooling group (Table 1). Figure 1 shows the temperature curves of each patient in both groups. The median duration within target temperature range was 19 of the 24 hours (interquartile range (IQR), 16-20) for the endovascular group versus 10 hours (IQR, 7-15) for the surface cooling group $(P=.0001)$. Median time from initiation of the cooling protocol to target temperature was 4 hours (240 minutes) (IQR, 2.8-6.2 hours) in the endovascular group versus 4.5 hours (270 minutes) (IQR, 3-6.5 hours) in the surface group $(P=\mathrm{NS})$. All patients in the endovascular group reached target temperature, but 
TABLE 1: Baseline characteristics and adverse events during the first week in the endovascular- $(N=26)$ and surface-cooled groups $(N=15)$.

\begin{tabular}{|c|c|c|c|}
\hline Characteristic/adverse event & Endovascular number $(\%)$ & Surface number $(\%)$ & $P$ value $^{\mathrm{a}}$ \\
\hline \multicolumn{4}{|l|}{ Baseline characteristic } \\
\hline Mean age (years) & $63 \pm 17$ & $58 \pm 15$ & .28 \\
\hline Sex (males) & $18(69 \%)$ & $12(80 \%)$ & .22 \\
\hline Mean weight $(\mathrm{kg})$ & $82 \pm 21$ & $95 \pm 37$ & .15 \\
\hline Duration of the arrest (min) & 31 & 24 & .13 \\
\hline Ventricular fibrillation arrests & $12(46 \%)$ & $1(7 \%)$ & .008 \\
\hline Median time from arrest to initiation of cooling protocol (min) & 277 & 481 & .16 \\
\hline Median Bladder T at initiation of cooling $\left({ }^{\circ} \mathrm{C}\right)$ & 36.4 & 36.4 & .76 \\
\hline Renal failure on admission ${ }^{\mathrm{b}}$ & $10(38 \%)$ & $10(67 \%)$ & .06 \\
\hline \multicolumn{4}{|l|}{ Adverse Events } \\
\hline Hypotension $^{c}$ & $10(38 \%)$ & $4(27 \%)$ & .21 \\
\hline Bradycardia $^{\mathrm{d}}$ & $18(69 \%)$ & $8(53 \%)$ & .16 \\
\hline Other arrhythmias & $6(23 \%)$ & $4(27 \%)$ & .28 \\
\hline New infection in 1st week & $16(62 \%)$ & $8(53 \%)$ & .23 \\
\hline Pneumonia & $14(54 \%)$ & $7(47 \%)$ & .23 \\
\hline Sepsis & $1(4 \%)$ & $0(0 \%)$ & .63 \\
\hline Pancreatitis & $0(0 \%)$ & $0(0 \%)$ & - \\
\hline Renal failure 1st week ${ }^{\mathrm{e}}$ & $6(23 \%)$ & $4(27 \%)$ & .25 \\
\hline Hemodialysis & $1(4 \%)$ & $1(7 \%)^{\mathrm{f}}$ & .48 \\
\hline Coagulopathy ${ }^{\mathrm{g}}$ & $6(23 \%)$ & $4(27 \%)$ & .77 \\
\hline Groin hematoma & $0(0 \%)$ & N/A & - \\
\hline Skin injury & $0(0 \%)$ & $0(0 \%)$ & - \\
\hline Transfusion $\mathrm{pRBC}$ & $6(23 \%)$ & $4(27 \%)$ & .28 \\
\hline Transfusion of platelets & $1(4 \%)$ & $0(0 \%)$ & .63 \\
\hline Seizures $^{\mathrm{h}}$ & $2(8 \%)$ & $2(13 \%)$ & .33 \\
\hline Deep venous thrombosis ${ }^{\mathrm{i}}$ & $3(12 \%)$ & $1(7 \%)$ & .38 \\
\hline Pulmonary embolism & $1(4 \%)$ & $0(0 \%)$ & .63 \\
\hline
\end{tabular}

${ }^{a} P$ values calculated from Fisher's exact test and Wilcoxon rank-sum test; ${ }^{b}$ creatinine $\geq 1.5 \mathrm{mg} / \mathrm{dL}$; ${ }^{c}$ mean arterial pressure $<70 \mathrm{~mm} \mathrm{Hg}$ and requiring pressors;

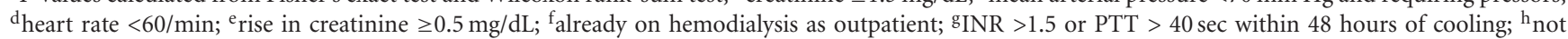
including clinical evidence of status myoclonus.

one patient in the surface group never did. Three patients $(12 \%)$ in the endovascular group had temperatures below $32^{\circ} \mathrm{C}$ for more than one hour, compared to $7(47 \%)$ in the surface group $(P=.022)$. However, one of the endovascular patients was initially treated with surface cooling and started off below target temperature. Median time from actual physical insertion of the catheter to target temperature was 114 minutes (IQR, 30-199). The frequency of predefined adverse events related to cooling or cooling technology in the first week was similar in both groups (Table 1). There was a trend towards more frequent hypotension and arrhythmias in the endovascular group. No catheter-related procedural complications and no skin injuries were observed. Mortality was $62 \%(16 / 26)$ for the endovascular group and $73 \%$ (11/15) for the surface group. Twenty-six percent of the patients in the endovascular group were discharged home, $4 \%$ to a rehabilitation facility, and $8 \%$ to a nursing home.
Of the surface group, $7 \%$ went home, $7 \%$ went to a rehabilitation facility, and 13\% went to a nursing home.

\section{Discussion}

The results of this study demonstrate that temperature control using the Celsius Control System Innercool catheter is more accurate in keeping patients in the target temperature range than surface cooling with ice bags and cooling blankets. Two similar studies also found that endovascular cooling is superior to surface cooling in maintaining a target temperature $[15,16]$. Further, another study found that unintentional overcooling occurred in the majority of patients (63\%) treated with ice bags and conventional cooling blankets [17]. In our study this occurred in $12 \%$ of the patients in the endovascular group and $47 \%$ of the surface group. Accurate temperature control is important 
as body temperatures above target temperature may offer less neuroprotection and body temperatures below target may produce more pronounced side effects. The observed trend of higher frequencies of hypotension and bradycardia in our endovascular group probably may reflect an overall longer duration of lower body temperatures in this group during the cooling maintenance phase compared to the surface group but it may also be related to the position of the catheter in relative proximity to the heart.

We felt that a pragmatic comparison between the two cooling methods in our study was the most appropriate and decided to compare them from the time point that it was decided to initiate the cooling protocol to reaching target temperature, hence including the organizational time required to implement each one of the two cooling techniques. After all, if one technique cools patients faster than another, but requires more time to initiate because of process issues, its apparent benefit may be negated. We observed a trend to reach target temperatures faster in the endovascularcooled group despite the additional time required to move the patient to an intensive care unit bed or the cardiac catheterization laboratory prior to insertion of the catheter. Furthermore, sometimes a neurointensivist traveled from home to place the catheter. Adjunct cooling methods, such as ice-cooled intravenous fluid infusions, might have decreased the time to target temperature in both groups but were not used during the study period [18].

There was a trend towards better neurologic outcomes in the endovascular group. This finding may be based on imbalances in baseline characteristics and should not be interpreted as a suggestion of better clinical outcomes with endovascular cooling. Further, with increasing familiarity with the use of hypothermia at our institution, the time from arrest to initiation of cooling protocol decreased over time and tended to be less in the endovascular group. Thus, data of this study cannot be used to compare the clinical benefit of endovascular with surface cooling.

While this study did not specifically measure nursing satisfaction with the two cooling techniques, part of the reason we changed to endovascular cooling from surface cooling was that the nursing staff found the surface method very labor intensive. The application and removal of the ice bags and cooling blankets to maintain the target temperature was difficult and time consuming for the nursing staff. Despite the nurses efforts some patients would remain above or below the target range. The endovascular method, once in place, frees the nurse to focus on other duties, because temperature is automatically maintained in target range. Both techniques require an additional piece of equipment to be in the patient's room and make transporting a patient more cumbersome. Special attention must be paid by the nurse when turning or transporting the endovascularly cooled patient as the patient's leg must be kept straight on the insertion side. For the physician the endovascular technique is more labor intensive, and for those unfamiliar with the device it will require procedural training for placement.

Limitations of this study include its nonrandomized design and imbalances in the baseline characteristics between the two groups; however, we intended to compare performance of cooling technologies and not patient outcome. It is possible that newer, more sophisticated surface cooling technology, such as adhesive surface devices with patient temperature feedback to computer-controlled temperature management systems, performs even better than endovascular cooling techniques. Similarly, there may also be differences in performance among various commercially available cooling catheters. Other studies have reported effective temperature control and safety with the Icy catheter and CoolGard system $[13,14]$. Finally we did not do a cost comparison between the two groups.

\section{Conclusions}

Endovascular cooling to mild-to-moderate hypothermia is feasible in comatose postcardiac arrest patients. When compared with conventional surface cooling it is more accurate in maintaining target temperature, but it is not faster in terms of reaching target temperature, because of time lost to logistic issued associated with catheter insertion and setting up the cooling console. Further studies are needed to assess if differences in cooling accuracy translate into better clinical outcomes.

\section{Disclosure}

The authors have reported no conflicts of interest. The use of the Celsius Control System Innercool catheter for cooling postcardiac arrest patients is offlabel usage of the device.

\section{Appendix}

\section{A. Stanford Therapeutic Hypothermia after Cardiac Arrest Protocol}

This is adapted from the University of California San Francisco hypothermia after cardiac arrest cooling protocol with permission.

\section{A.1. Inclusion Criteria}

(1) Age 18 years or older.

(2) Women must be over 50 or have a negative pregnancy test.

(3) Cardiac arrest with return of normal sinus rhythm.

(4) Persistent coma as evidenced by no eye opening to pain after resuscitation (no waiting period required).

(5) Blood pressure can be maintained at least at $90 \mathrm{~mm}$ $\mathrm{Hg}$ systolic either spontaneously or with fluid and pressor (no aortic balloon pump unless approved by cardiology).

(6) Modified Rankin scale 0-2 prior to the arrest. 


\section{A.2. Exclusion Criteria}

(1) Any other overt reason to be comatose (e.g., sedating drugs, drug overdose, status epilepticus).

(2) Pregnancy.

(3) A known terminal illness preceding the arrest.

(4) Known pre-existing coagulopathy or bleeding.

(5) Pre-existing do not intubate code status and patient not intubated as part of the resuscitation efforts.

\section{A.3. Protocol (Goal Temperature $33.0^{\circ} \mathrm{C}$ to Be Achieved as Soon} as Possible)

(1) Hypothermia should be initiated as quickly as possible. For out-of-hospital arrests the ED attending in conjunction with the neurocritical care/stroke team will make the decision to implement the protocol. For in-hospital arrests, the neurocritical care/stroke team in conjunction with the CCU or ICU fellow in charge of the patient will make the decision.

(2) Page Neurology resident in house for immediate neurological assessment prior to pharmacologic paralysis. Do not delay initiation of cooling pending assessment.

(3) Immediately place ice bags under the armpits, next to the neck, on the torso and limbs.

(4) Temperature sensing Foley catheter should be placed, otherwise rectal or tympanic temperatures should be used (in that order) until Foley placement.

(5) Two cooling blankets should be used, one under and one over the patient. Both should be set to maximum cooling.

(6) The room thermostat should be turned off.

(7) Administer midazolam and fentanyl for sedation or other sedatives as ordered by the primary team.

(8) Once sedation is started and effective, give a vecuronium bolus, then continuous drip for paralysis or other paralyzing agent as ordered by the primary team. Titrate the drip to keep 1-2/4 twitches on train of four.

(9) Patients should be on sliding scale insulin to maintain glucose between $151-200 \mathrm{mg} / \mathrm{dL}$, daily aspirin, pressors to maintain blood pressure, and any antiarrhythmics as necessary. Place nasogastric tube for meds.

(10) Patients may receive other cardiac interventions including systemic thrombolysis, anticoagulation, and urgent cardiac catheterization interventions as needed. Hypothermia should proceed concurrent with these interventions.

(11) Once the patient reaches $34^{\circ} \mathrm{C}$, remove the ice packs and top cooling blanket if necessary. The goal is for the patient's temperature to remain between 32$34^{\circ} \mathrm{C}$.
(12) Begin passive rewarming 24 hours after the beginning of cooling (not 24 hours after target temperature is reached). The goal is to reach $36.5-37^{\circ} \mathrm{C}$ (If temperature increases over $37.5^{\circ} \mathrm{C}$ restart cooling blankets).

(a) Turn room thermostat up to normal.

(b) Discontinue cooling blankets.

(c) May use regular blankets.

(d) Do not use warm air blankets unless temperature not at $36^{\circ} \mathrm{C}$ after 12 hours of passive rewarming.

(13) Paralysis, then sedation, may be discontinued after rewarming to $36.5^{\circ} \mathrm{C}$.

\section{A.4. Supplement-Use of Endovascular Cooling Catheter.}

(1) The neurocritical care/stroke team should be paged as part of the standard protocol for considering hypothermia following cardiac arrest. The neurocritical care/stroke team will evaluate the patient for endovascular cooling.

(2) External cooling should be initiated immediately as per the standard protocol, pending evaluation for endovascular cooling. Patient must be able to tolerate large-bore catheter (as large as $14 \mathrm{~F}$ ) into the inferior vena cava via a femoral vein.

(3) If possible inform family members of the procedure and its risks and benefits and make a note of this in the chart.

(4) Equipment needed for endovascular cooling:
(a) innercool console,
(b) console-related equipment,
(c) cooling catheter equipment.

(5) The neurocritical care/stroke team will place the endovascular catheter. Cooling will then be initiated using the innercool console to a target temperature of $33^{\circ} \mathrm{C}$ for 24 hours. All external cooling elements may be discontinued. Rewarming will take place passively over 12 hours to a target temperature of $36.5-37^{\circ} \mathrm{C}$.

(6) An abdominal X-ray should be obtained following placement of the catheter, but initiation of the endovascular cooling should not be delayed pending the result of the study.

(7) Sedation, paralytics and mechanical ventilation should be employed as part of the standard hypothermia after cardiac arrest protocol.

(8) Patients may receive other cardiac interventions, including systemic thrombolysis, anticoagulation and urgent cardiac intervention as needed.

(9) The neurocritical care team will remove the catheter at the end of the 24-hour cooling period.

(10) Start heparin subcutaneously 5000 unit q 12 hours, 12 hours after the catheter is removed. 


\section{References}

[1] W. Rosamond, K. Flegal, G. Friday et al., "Heart disease and stroke statistics—2007 update: a report from the American Heart Association Statistics Committee and Stroke Statistics Subcommittee," Circulation, vol. 115, no. 5, pp. e69-e171, 2007.

[2] Z. J. Zheng, J. B. Croft, W. H. Giles, and G. A. Mensah, "Sudden cardiac death in the United States, 1989 to 1998," Circulation, vol. 104, no. 18, pp. 2158-2163, 2001.

[3] M. F. Hazinski, A. H. Idris, R. E. Kerber et al., "Lay rescuer automated external defibrillator ("public access defibrillation") programs: lessons learned from an international multicenter trial advisory statement from the American Heart Association Emergency Cardiovascular Committee; the Council on Cardiopulmonary, Perioperative, and Critical Care; and the Council on Clinical Cardiology," Circulation, vol. 111, no. 24, pp. 3336-3340, 2005.

[4] K. A. Ballew and J. T. Philbrick, "Causes of variation in reported in-hospital CPR survival: a critical review," Resuscitation, vol. 30, no. 3, pp. 203-215, 1995.

[5] Hypothermia after Cardiac Arrest Study Group, "Mild therapeutic hypothermia to improve the neurologic outcome after cardiac arrest," The New England Journal of Medicine, vol. 346, pp. 549-556, 2002.

[6] S. A. Bernard, T. W. Gray, M. D. Buist et al., "Treatment of comatose survivors of out-of-hospital cardiac arrest with induced hypothermia," The New England Journal of Medicine, vol. 346, no. 8, pp. 557-563, 2002.

[7] "American Heart Association guidelines for cardiopulmonary resuscitation and emergency cardiovascular care: part 7.5: postresuscitation support," Circulation, vol. 112, pp. IV-84IV-88, 2005.

[8] M. A. Perberdy, C. W. Callaway, R. W. Neumar et al., "Part 9: post-cardiac arrest care: 2010 American Heart Association guidelines for cardiopulmonary resuscitation and emergency cardiovascular care," Circulation, vol. 122, pp. S768-S786, 2010.

[9] B. S. Abella, J. W. Rhee, K. N. Huang, T. L. Vanden Hoek, and L. B. Becker, "Induced hypothermia is underused after resuscitation from cardiac arrest: a current practice survey," Resuscitation, vol. 64, no. 2, pp. 181-186, 2005.

[10] R. M. Merchant, J. Soar, M. B. Skrifvars et al., "Therapeutic hypothermia utilization among physicians after resuscitation from cardiac arrest," Critical Care Medicine, vol. 34, no. 7, pp. 1935-1940, 2006.

[11] K. Kuboyama, P. Safar, A. Radovsky, S. A. Tisherman, S. W. Stezoski, and H. Alexander, "Delay in cooling negates the beneficial effect of mild resuscitative cerebral hypothermia after cardiac arrest in dogs: a prospective, randomized study," Critical Care Medicine, vol. 21, no. 9, pp. 1348-1358, 1993.

[12] V. Weinrauch, P. Safar, S. Tisherman, K. Kuboyama, and A. Radovsky, "Beneficial effect of mild hypothermia and detrimental effect of deep hypothermia after cardiac arrest in dogs," Stroke, vol. 23, no. 10, pp. 1454-1462, 1992.

[13] F. M. Al-Senani, C. Graffagnino, J. C. Grotta et al., "A prospective, multicenter pilot study to evaluate the feasibility and safety of using the CoolGard System and Icy catheter following cardiac arrest," Resuscitation, vol. 62, no. 2, pp. 143150, 2004.

[14] M. Holzer, M. Müllner, F. Sterz et al., "Efficacy and safety of endovascular cooling after cardiac arrest: cohort study and Bayesian approach," Stroke, vol. 37, no. 7, pp. 1792-1797, 2006.
[15] A. C. Flint, J. C. Hemphill, and D. C. Bonovich, "Therapeutic hypothermia after cardiac arrest: performance characteristics and safety of surface cooling with or without endovascular cooling," Neurocritical Care, vol. 7, no. 2, pp. 109-118, 2007.

[16] M. A. Gillies, R. Pratt, C. Whiteley, J. Borg, R. J. Beale, and S. M. Tibby, "Therapeutic hypothermia after cardiac arrest: a retrospective comparison between surface cooling and endovascular cooling," Resuscitation, vol. 81, pp. 1117-1122, 2010.

[17] R. M. Merchant, B. S. Abella, M. A. Peberdy et al., "Therapeutic hypothermia after cardiac arrest: unintentional overcooling is common using ice packs and conventional cooling blankets," Critical Care Medicine, vol. 34, no. 12, pp. S490-S494, 2006.

[18] A. Kliegel, A. Janata, C. Wandaller et al., "Cold infusions alone are effective for induction of therapeutic hypothermia but do not keep patients cool after cardiac arrest," Resuscitation, vol. 73, no. 1, pp. 46-53, 2007. 


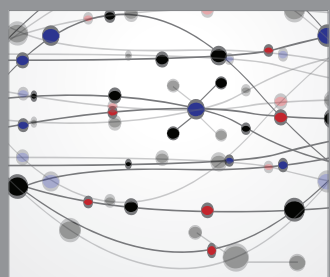

The Scientific World Journal
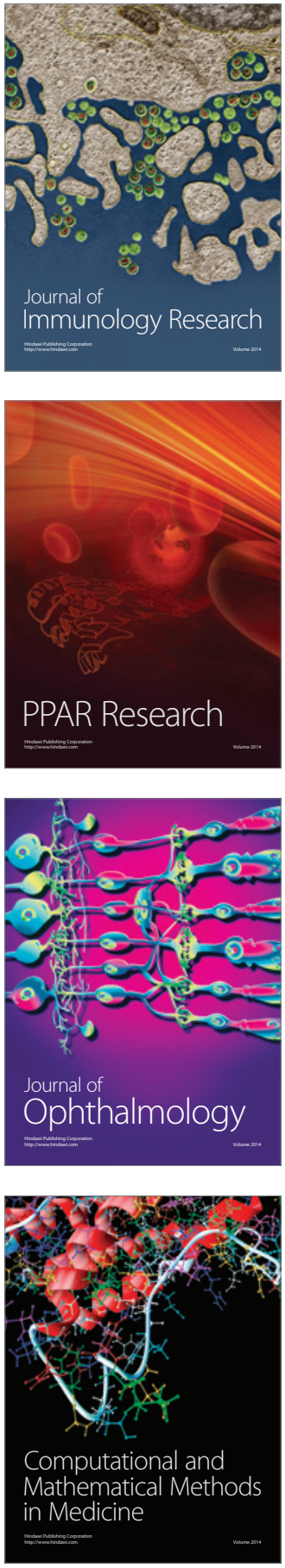

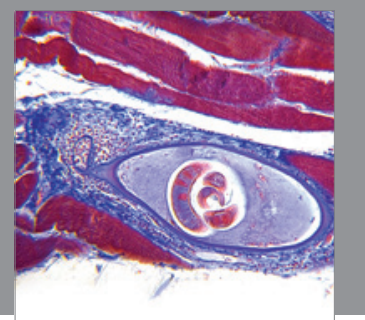

Gastroenterology

Research and Practice
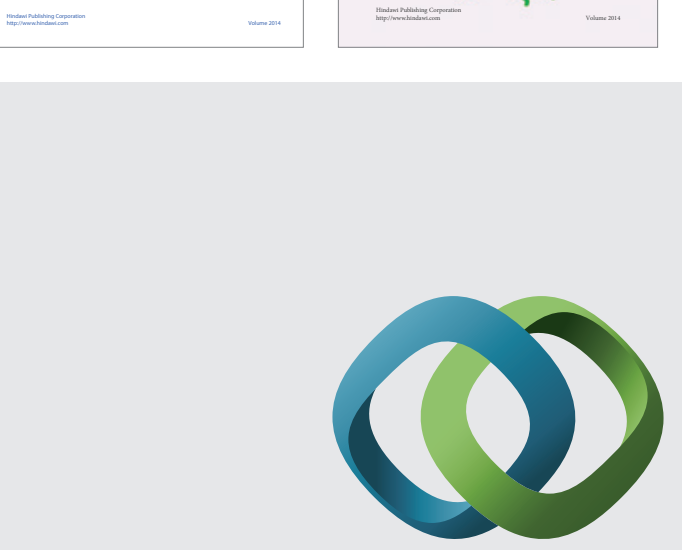

\section{Hindawi}

Submit your manuscripts at

http://www.hindawi.com
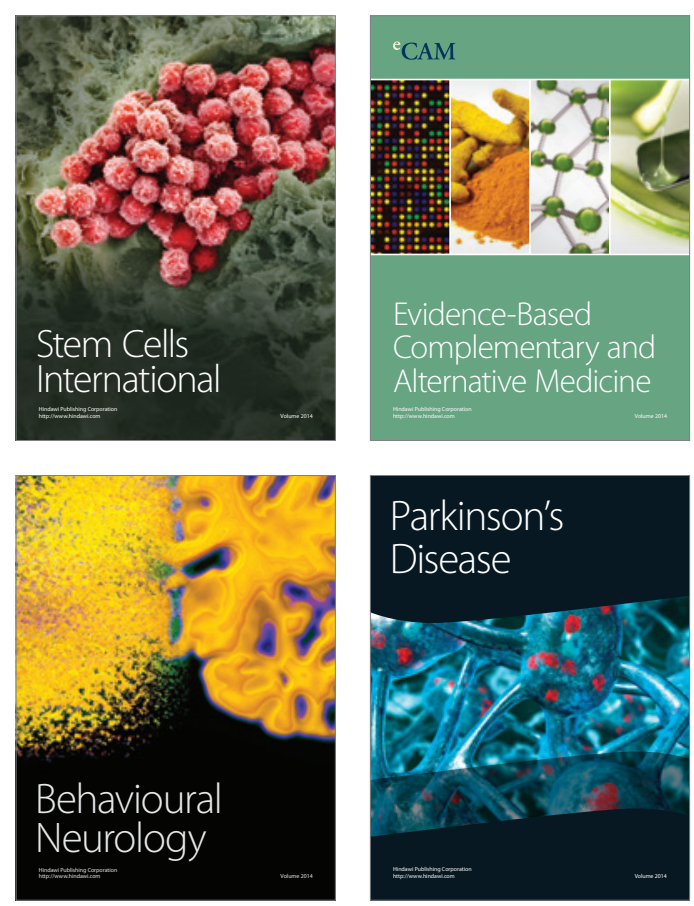

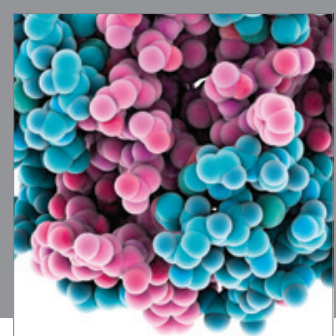

Journal of
Diabetes Research

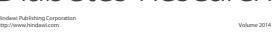

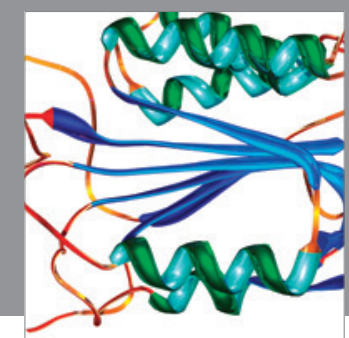

Disease Markers
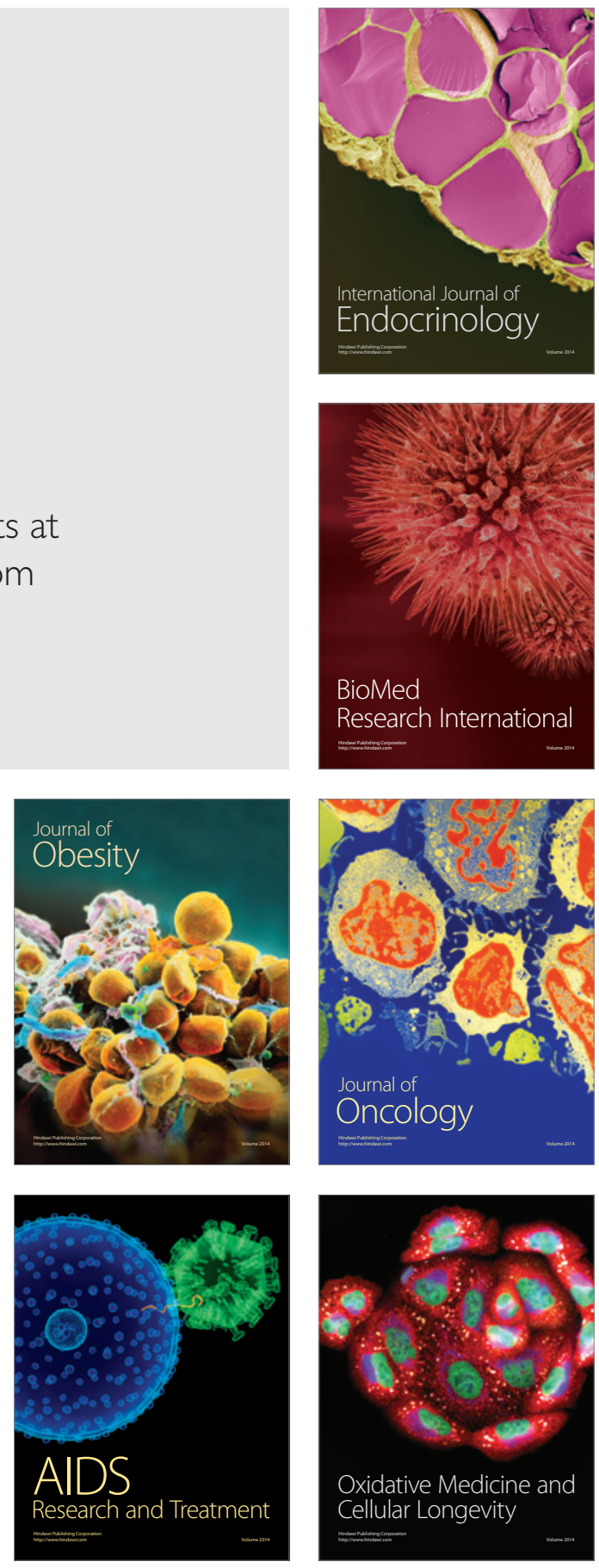